\title{
COMPARATIVE EVALUATION OF MULTIPLE STAINING TECHNIQUES FOR IDENTIFICATION OF DIFFERENT DEVELOPMENTAL STAGES OF ACANTHAMOEBA AND NAEGLERIA
}

By

\author{
HANAN EM ELDEEK ${ }^{*}$, RASHA AH ATTIA, MARTINA M NAGEEB, \\ And ATEF A SAKLA \\ Department of Medical Parasitology, Faculty of Medicine, \\ Assiut University, Assiut, Egypt. \\ ( ${ }^{*}$ Correspondence: hananhmo@yahoo.com, Mobile 01009092470)
}

\begin{abstract}
Potentially pathogenic free-living amoebae (FLA) are unique protozoa that present a healthcare challenge. The appropriate choice of staining technique is crucial to improving the efficiency of microscopic diagnosis of FLA isolated from environmental as well as clinical samples. This study aimed to evaluate different staining techniques for morphological identification of Acanthamoeba and Naegleria isolated from tap water samples in different localities in Assiut City, Egypt. A multi-attribute evaluation was performed to compare these techniques considering staining quality, ease of performance, cost and time of each procedure. Seven staining techniques were used including Lugol's iodine and lactophenol cotton blue as temporary stains, with Giemsa, trichrome, iron hematoxylin, acid-fast and Gram stains as permanent stains. Both lactophenol cotton blue and Lugol's iodine showed good structural details of different stages of FLA and good contrast at a very short time and reasonable cost, followed by Giemsa. Both trichrome and iron hematoxylin stains gave comparable staining quality but at a longer time with more complex staining procedures and higher cost. Both modified acid-fast and Gram stains didn't provide sufficient staining quality to identify morphological details of FLA. According to the multi-attribute evaluation, both Lugol's iodine and lactophenol cotton blue achieved the highest rank (92.85\%) followed by Giemsa and Gram stains $(64.28 \%)$, modified acid-fast stain $(57.14 \%)$, while iron hematoxylin and Masson's trichrome had the last rank (46.42\%).
\end{abstract}

Keywords Acanthamoeba, Naegleria, trophozoite, cyst, stains.

\section{Introduction}

Free-living amoebae (FLA) are a large diverse group of amoebic protozoa that exist in a wide range of habitats where they feed on bacteria, fungi, algae, and small organic particles. Yet, some of them can become tissue feeders and facultative pathogens to humans (Khurana et al, 2015). Water plays an important role in the transfer of FLA as they detected in numerous natural and manmade aquatic environments (Abdul Majid $e t$ $a l, 2017)$ including sea water, drinking water, bottled mineral water, swimming pools, sewage, eyewash solutions, contact lenses solutions, dialysis units, and dental treatment units (Visvesvara et al, 2007; Balczun and Scheid, 2017). The potentially pathogenic FLA are able to survive and proliferate in the environment independently and their cysts are extremely resistant to harsh condi- tions due to the process of encystment (Schuster and Visvesvara, 2004; Khan, 2006; Teixera et al, 2009).

Acanthamoeba and Naegleria are the most common amphizoic FLA that relate to man and animal diseases (Ozcelik et al, 2012). Acanthamoeba causes the fatal disease granulomatous amoebic encephalitis (GAE) in immunocompromised patients and visionthreatening keratitis in immunocompetent people while Naegleria fowleri, causes an acute and rapidly fatal primary amoebic meningoencephalitis (PAM) affecting healthy people. Marked improvement of outcome of infections can be achieved by early diagnosis, coupled with effective specific therapy (Król-Turmińska and Olender, 2017).

Conclusive diagnosis of GAE, PAM and Acanthamoeba keratitis (AK) depend upon the morphological identification of the tro- 
phozoite or cyst, either directly or after in vitro cultivation of contaminated samples. It is restricted by false-negative results due to the lack of technical expertise (Boggild et al, 2009). Wet mount technique has the ability to reveal the motility of the trophozoite but the ill-defined transparent internal structures of cysts or trophozoites with the presence of other microorganisms (growing together on the agar surface as bacteria or fungi) leads to misdiagnosis of these cases (El-Sayed and Hikal, 2015). Therefore staining is essential to offer full structural details with a better observation of the morphology due to the color contrast and stained cellular organelles (Ithoi et al, 2011).

Several staining procedures including temporary and permanent stains are used for the identification of amoebae in different clinical and environmental samples including iodine wet mount stain, Gram, Gimenez, Giemsa, chlorazol black, hematoxylin-eosin, periodic acid-Schiff, Masson's trichrome, iron hematoxylin, lactophenol cotton blue, Field's stain, Gomori methenamine silver, calcofluor white (CFW), and acridine orange stains (Pirehma et al, 1999; Shen et al, 2005; Thomas et al, 2011; El-Sayed and Hikal, 2015). Some of them are time-consuming so most laboratories choose one of the shorter staining procedures for routine diagnostic work.

The present study was designed to evaluate the use of different staining techniques for morphological identification of the trophozoite and cyst stages of Acanthamoeba and Naegleria isolated from tap water samples in different localities in Assiut City, Egypt. A systematic multi-attribute evaluation was performed to compare these techniques in view of their staining quality and other properties.

\section{Materials and Methods}

Isolation and preparation of amoebae: Several isolates of Acanthamoeba and Naegleria, obtained from tap water samples in different localities in Assiut City (Assiut Governorate, Egypt), were used. For each tap water sample, isolation was carried out by filtration of one-liter water through a $0.45-\mu \mathrm{m}$ pore-size filter and inoculation onto non-nutrient agar plates pre-seeded with heat-deactivated E.coli. The Plates were incubated at $38^{\circ} \mathrm{C}$ and observed daily for the presence of different genera of amoebae (Init et al, 2010). Examination of amoebic growth was carried out for 10 days using light and inverted microscope at $(\times 400)$. The agar plate culture containing amebae was flooded with $5 \mathrm{~mL}$ of Page amoeba saline (PAS) solution and pipetted to remove the trophozoites and cysts from the agar surface. The suspension was then transferred into a tube, centrifuged at $2500 \mathrm{rpm}$ for 10 minutes and the supernatant was discarded (Ithoi et al, 2011; Ozcelik et al, 2012; El-Sayed and Hikal, 2015). This step was repeated three times in order to remove additional agar and to isolate the highest amount of amoebae (Ozcelik et al, 2012). The clean sediment was then used for further microscopic examination, photographing and staining procedures.

Acanthamoeba and Naegleria were identified at genus level, based on the morphological features of cysts and trophozoites, according to key of Page (1988). Enflagellation experiment was done to verify the presence of Naegleria spp. (Visvesvara, 1999).

Seven stains were used and compared in the present study, including two temporary (Lugol's iodine and lactophenol cotton blue) and five permanent stains (Giemsa, Iron hematoxylin, Masson's trichrome, Modified Acid-fast, and Gram stains).

1- Temporary staining (wet-mount staining) Lugol's iodine and lactophenol cotton blue stains were used. Lugol's iodine solution was prepared by adding $10 \mathrm{~g}$ potassium iodide to $100 \mathrm{~mL}$ distilled water; when dissolved, $5 \mathrm{~g}$ iodine crystals were added. Then the solution was filtered and stored in a tightly stoppered bottle (Gracia, 2007). Lactophenol cotton blue, ready to use, was purchased from Sigma-Aldrich (Fluka, SigmaAldrich product no. 61335) and used accord- 
ing to the manufacturer's instructions. The wet mount staining was set by spreading out two drops $(50 \mu \mathrm{L})$ of amoebae sediment on a clean glass slide. A drop of the stain was then added, and a coverslip was immediately applied to enclose the material. Then the stained slides were examined under a light microscope (Olympus CHA, Japan) at a magnification of $\times 400$ followed by $\times 1000$ and photographed for permanent record.

2- Permanent staining: For the preparation of a permanent-stained smear, approximately $50 \mu \mathrm{L}$ (two drops) of the previously prepared clean sediment was dropped on a glass slide and spread to make a smear. It was dried in a moist chamber at $37^{\circ} \mathrm{C}$ for half an hour and then fixed in absolute methanol for approximately 5 minutes (Ithoi et al, 2011). The fixed smears were subsequently stained with the following staining techniques: iGiemsa stain: The fixed smears were stained with ready-to-use Giemsa solution (SigmaAldrich, Switzerland) after dilution at a concentration of 1: $5 \mathrm{~mL}$ of distilled water in a staining container for 20 minutes. The slides were then rinsed with water and let to airdry at room temperature for microscopic examination (El-Sayed and Hikal, 2015).

ii-Iron hematoxylin stain: Briefly, the fixed smears were placed in $70 \%$ ethanol for 5 minutes, washed in running tap water for 10 minutes, placed in iron hematoxylin working solution (mixed equal volumes of solutions A and B purchased from Electron Microscopy Sciences, Hatfield, Pennsylvania, USA) for 4 to 5 minutes, followed by another wash in running tap water for 10 minutes. The slides were then placed in $70 \%$ ethanol followed by $95 \%$ ethanol for 5 minutes each and double changes of $100 \%$ ethanol for 5 minutes each. Finally, the slides were placed in two changes of xylene for 5 minutes each (Garcia, 2007).

iii- Masson's trichrome stain: The fixed smears were stained with ready-to-use Masson's trichrome kit (Alpha Lab Diagnostic, Egypt). According to the manufacturer's instructions; the fixed smears were stained with Biebrich Scarlet-acid fuchsin solution for 10 minutes and washed in running warm tap water for 10 minutes. They were differentiated in a phosphomolybdic-phosphotungstic acid solution for 15 minutes and washed in distilled water. Subsequently, they have transferred directly to Aniline blue solution for 5 minutes and rinsed briefly in distilled water. Then they were differentiated in $1 \%$ acetic acid solution for 2-5 minutes and washed in distilled water. Finally, they were dehydrated through $95 \%$ ethyl alcohol, absolute ethyl alcohol and cleared in xylene. iv- Modified Acid-fast stain (Modified Kinyoun's Acid-Fast stain): The fixed smears were stained with ready-to-use acid-fast stain kit (Egyptian Diagnostic Media EDM, Egypt). According to the manufacturer's instructions; the slides were flooded with carbol fuchsin and let to be stained for 5 minutes, rinsed briefly for 5 seconds with $50 \%$ ethanol and then rinsed thoroughly with water. The slides were decolorized with a decolorizing solution (hydrochloric acid in ethanol) for 2 minutes or until no more color was observed from the slide. Then, the slides were rinsed with water. Finally, they were counterstained with methylene blue for 1 minute, rinsed with water and allowed to be air-dried.

v- Gram stain: The fixed smears were stained with ready-to-use Gram stain kit (Egyptian Diagnostic Media EDM, Egypt). According to the manufacturer's instructions; the slides were stained with crystal violet for 1 minute and washed with tap water. Then, Lugol's iodine was applied for 1 minute and rinsed again with tap water. The slides were then subjected to Gram's decolorizing solution for 30 seconds and rinsed with tap water. Finally, they were stained with Safranin for one minute, rinsed again and then air-dried.

All permanently stained slides were examined microscopically (Olympus CHA, Japan) at a magnification of $x 400$ followed by $\mathrm{x} 1000$, then photographed for permanent record. 
Assessment of different staining techniques The current staining techniques were evaluated according to multiple technical points, chiefly, staining quality, including color and contrast, clarity of morphological details, and differentiation between nuclei and cytoplasm (El-Sayed and Hikal, 2015), and other features of these techniques regarding ease of performance, time needed to finish the procedure, and its cost (the material and reagent costs were calculated for each procedure). A multi-attribute assessment method was adopted (MacPherson and McQueen 1993) to compare these staining techniques. This method recognizes, specifies, and then combines the different variables to elaborate numerical ranking of the techniques. The attributes were given a rank order from one to seven, with one being less desirable and seven being most desirable. This approach assumes that each attribute is of equal importance.

\section{Results}

Morphological characterization: The characters of trophozoite and cyst stages of Acanthamoeba and Naegleria stained with different staining techniques are shown in Figs. 1-7. Trophozoites of Acanthamoeba were easily identified by the unique and characteristic presence of fine, tapering, thorn-like acanthopodia that arise from the surface of the body. The trophozoites of all isolates were irregular in shape and ranged in size from 25 to $50 \mu \mathrm{m}$. They were uninucleate, although binucleated trophozoites were also encountered, and the nucleus had a centrally placed large nucleolus. Endoplasm contained numerous food vacuoles, and a contractile vacuole (Figs.1a, 2a, 3a,b, 4a, 5a, 6a,b, \&7a).
Cysts of Acanthamoeba were doublewalled and ranged in size from 11 to $25 \mu \mathrm{m}$. The outer cyst wall, the ectocyst, was wrinkled with folds in most species. The inner cyst wall, the endocyst, varied in shape; stellate, polygonal, oval, or spherical depending upon the species. Each cyst had a single nucleus with a centrally placed dense nucleolus (Figs.1b, 2b, 3c, 4b, 5b, 6c, \& 7b).

Naegleria trophozoites ranged in size from 15 to $25 \mu \mathrm{m}$. Each had a single nucleus with a prominent nucleolus. Endoplasm contained numerous food vacuoles and, at the posterior end, contractile vacuole. Locomotion was achieved by the use of lobopodia; hemispherical bulges at the anterior end (Figs.1c, 2c, 3d, 4c, 5c, 6d, \& 7c).

Naegleria cysts were spherical and double-walled. Each with a thick endocyst and a closely opposed thin ectocyst, measuring $8-12 \mu \mathrm{m}$ in diameter (Figs.1d, 2d, $3 \mathrm{e}, 4 \mathrm{~d}, 5 \mathrm{~d}, 6 \mathrm{e}, \& 7 \mathrm{~d})$.

Regarding staining quality, Lugol's iodine and lactophenol cotton blue stains were superior to other used stains followed by Giemsa, iron hematoxylin and Masson's trichrome stains, while Gram and modified acid-fast stains ranked last

Multi-attribute evaluation of the seven different stains used in this study showed that both Lugol's iodine and lactophenol cotton blue stains achieved the highest rank $(92.85 \%)$ followed by both Gram and Giemsa stains with a rank of $64.28 \%$, followed by modified acid-fast stain $(57.14 \%)$ while both iron hematoxylin and Masson's trichrome stains had the last rank (46.42\%)

The details were given in tables $(1 \& 2)$ and figures $(1,2,3,4,5,6 \& 7)$. 
Table 1: Comparison of staining quality of different staining techniques to identify trophozoite and cyst stages of Acanthamoeba and Naegleria.

\begin{tabular}{|c|c|c|c|c|c|c|c|}
\hline \multirow[t]{3}{*}{ Stains } & \multirow{3}{*}{$\begin{array}{l}\text { Color \& } \\
\text { Contrast }\end{array}$} & \multicolumn{4}{|c|}{ Acanthamoeba } & \multicolumn{2}{|c|}{ Naegleria } \\
\hline & & \multicolumn{2}{|c|}{$\begin{array}{c}\text { Trophozoite } \\
\end{array}$} & \multicolumn{2}{|r|}{ Cyst } & Trophozoite & Cyst \\
\hline & & Differentiation* & Acanthopodia & Nucleus & Walls & $\begin{array}{l}\text { Differentia- } \\
\text { tion* }\end{array}$ & Nucleus \\
\hline $\begin{array}{l}\text { Lugol's } \\
\text { iodine }\end{array}$ & $\begin{array}{c}\text { Yellow } \\
+\end{array}$ & $\begin{array}{l}\text { Well differentia- } \\
\text { tion }\end{array}$ & Well recognized & $\begin{array}{l}\text { Well recog- } \\
\text { nized }\end{array}$ & Stained both walls & $\begin{array}{l}\text { Well differen- } \\
\text { tiation }\end{array}$ & $\begin{array}{l}\text { Well recog- } \\
\text { nized }\end{array}$ \\
\hline $\begin{array}{l}\text { Lactophenol } \\
\text { cotton blue }\end{array}$ & $\begin{array}{c}\text { Blue } \\
+\end{array}$ & $\begin{array}{l}\text { Well differentia- } \\
\text { tion }\end{array}$ & Well recognized & $\begin{array}{l}\text { Well recog- } \\
\text { nized }\end{array}$ & Stained both walls & $\begin{array}{l}\text { Well differen- } \\
\text { tiation }\end{array}$ & $\begin{array}{l}\text { Hardly } \\
\text { recognized }\end{array}$ \\
\hline Giemsa & $\begin{array}{c}\text { Blue } \\
+\end{array}$ & $\begin{array}{l}\text { Well differentia- } \\
\text { tion }\end{array}$ & Well recognized & $\begin{array}{l}\text { Not recog- } \\
\text { nized }\end{array}$ & Stained endocyst only & $\begin{array}{l}\text { Well differen- } \\
\text { tiation }\end{array}$ & $\begin{array}{l}\text { Hardly } \\
\text { recognized }\end{array}$ \\
\hline $\begin{array}{l}\text { Iron hema- } \\
\text { toxylin }\end{array}$ & $\begin{array}{c}\text { Grey } \\
\pm\end{array}$ & $\begin{array}{l}\text { Well differentia- } \\
\text { tion }\end{array}$ & $\begin{array}{l}\text { Well recog- } \\
\text { nized }\end{array}$ & $\begin{array}{l}\text { Not recog- } \\
\text { nized }\end{array}$ & Stained both walls & $\begin{array}{l}\text { Well differen- } \\
\text { tiation }\end{array}$ & $\begin{array}{l}\text { Well recog- } \\
\text { nized }\end{array}$ \\
\hline $\begin{array}{l}\text { Masson's } \\
\text { trichrome }\end{array}$ & $\begin{array}{c}\text { Purple } \\
+\end{array}$ & $\begin{array}{l}\text { Well differentia- } \\
\text { tion }\end{array}$ & Well recognized & $\begin{array}{l}\text { Not recog- } \\
\text { nized }\end{array}$ & $\begin{array}{c}\text { Stained endocyst } \\
\text { faintly stained ectocyst }\end{array}$ & $\begin{array}{l}\text { Well differen- } \\
\text { tiation }\end{array}$ & $\begin{array}{l}\text { Not recog- } \\
\text { nized }\end{array}$ \\
\hline $\begin{array}{l}\text { Modified } \\
\text { acid-fast }\end{array}$ & $\begin{array}{c}\text { Light blue } \\
+\end{array}$ & $\begin{array}{c}\text { Poor differentia- } \\
\text { tion }\end{array}$ & Well recognized & $\begin{array}{l}\text { Not recog- } \\
\text { nized }\end{array}$ & Stained both walls & $\begin{array}{l}\text { Poor differenti- } \\
\text { ation }\end{array}$ & $\begin{array}{l}\text { Not recog- } \\
\text { nized }\end{array}$ \\
\hline Gram & $\begin{array}{c}\text { Red } \\
+\end{array}$ & $\begin{array}{l}\text { Poor differentia- } \\
\text { tion }\end{array}$ & $\begin{array}{l}\text { Hardly recog- } \\
\text { nized }\end{array}$ & $\begin{array}{l}\text { Not recog- } \\
\text { nized }\end{array}$ & Stained endocyst only & $\begin{array}{l}\text { Well differen- } \\
\text { tiation }\end{array}$ & $\begin{array}{l}\text { Hardly } \\
\text { recognized }\end{array}$ \\
\hline
\end{tabular}

*differentiation between internal organelles especially nuclei and cytoplasm, ${ }^{+}$good contrast between the cyst or trophozoite stage and surrounding background and ${ }^{ \pm}$poor contrast between cyst or trophozoite stage and surrounding background.

Table 2: Multi-attribute assessment of different staining techniques for identification of the trophozoites and cysts of Acanthamoeba and Naegleria.

\begin{tabular}{|l|c|c|c|c|l|}
\hline \multirow{2}{*}{ Stains } & \multicolumn{3}{|c|}{ Ranking for the following attribute: } & \multirow{2}{*}{ Rank } \\
\cline { 2 - 5 } & Staining quality & Ease of handling & Time & Cost & \\
\hline Lugol's iodine & 7 & 7 & 7 & 5 & $26 / 28(92.85 \%)$ \\
\hline Lactophenol cotton blue & 6 & 7 & 7 & 6 & $26 / 28(92.85 \%)$ \\
\hline Giemsa & 5 & 6 & 4 & 3 & $18 / 28(64.28 \%)$ \\
\hline Gram & 3 & 4 & 6 & 5 & $18 / 28(64.28 \%)$ \\
\hline Modified acid-fast & 3 & 4 & 5 & 4 & $16 / 28(57.14 \%)$ \\
\hline Iron hematoxylin & 5 & 3 & 3 & 2 & $13 / 28(46.42 \%)$ \\
\hline Masson's trichrome & 5 & 3 & 3 & 2 & $13 / 28(46.42 \%)$ \\
\hline
\end{tabular}

\section{Discussion}

Although several immunological and molecular methods have been developed for identification of FLA, the detection of their different developmental stages via microscopic examination remains the gold standard conventional method for diagnosis in the related infections. Primarily, diagnosis is based upon the morphological identification of the trophozoites and/or cysts either directly or by using different stains. Thus, the appropriate choice of the staining technique was essential to ensure the accurate results (Martinez and Visvesvara, 1997; Guarner et al, 2007).

In the present study, a systematic multiattribute evaluation was performed to compare these techniques. The morphological identification of the different developmental stages of Acanthamoeba and Naegleria was done by detecting specific organelles inside the trophozoites, the shape of the cysts and the presence of acanthopodia or lobopodia.
It was observed that non-stained wet-mount preparation of FLA has the advantage of demonstrating the trophozoite motility. However, in this technique, the different stages of amoebae appear transparent, with poorly visualized internal structures, and even the individual organisms sometimes could't be identified. Moreover, the coexistence of bacteria and fungi, growing with the amoebae on the agar surface, may aggravate the difficulty of amoebae detection (Sharma et al, 2000; Yera et al, 2007; Ithoi et al, 2011). Thus, staining is important to provide a detailed structure of different stages of amoebae to facilitate their visualization and identification.

Nevertheless, the outcome of each staining technique is variable depending upon many factors such as the stain type, concentration of the used solutions, the time needed to complete the procedure as well as the affinity of the stain to show the cyst wall (El-Sayed and Hikal, 2015). 
In this study used the multiple staining techniques, and did a comprehensive multiattribute evaluation for these methods, comparing them upon the bases of their staining quality, the ease of performance, cost and time taken for each procedure. The used staining techniques were defined according to their stability into temporary and permanent stains.

The temporary stains, including Lugol's iodine and lactophenol cotton blue, stained the internal structures of trophozoites and cysts both in Acanthamoeba and Naegleria yellow and blue, respectively, and they have shown clear nuclei. The acanthopodia or lobopodia of trophozoites were stained the same as the corresponding cytoplasm. These stains showed good contrast with the surroundings making organisms clearly differentiated.

The present results agreed with Thomas and Kuriakose (1990) who used lactophenol cotton blue stain for diagnosis of Acanthamoeba keratitis. They stated that this stain is particularly well suited for Acanthamoeba cysts since the double-wall of the cysts stained dark blue with good contrast against the surroundings. However, their results also showed that the Acanthamoeba trophozoites took the diffuse blue appearance that could be mistaken with the surrounding cells. This finding contradicts with the present results.

In the present results also complied with those of Le Govic et al. (2018) who used Kop-color ${ }^{\circledR}$ (iodine containing stain) and lactophenol cotton blue stains to detect Acanthamoeba cysts in cultures from a case of AK. These authors recommended the use of this technique which could improve the efficiency of conventional diagnosis of AK.

Temporary stained wet mounts in the present study were a simple, rapid, and single-step procedure for identification of FLA. Moreover, they are inexpensive stains and readily available in any clinical laboratory with no need for special microscopy (Thomas and Kuriakose, 1990; Grossnik- laus et al, 2003). However, El-Sayed and Hikal (2015) stated that these preparations are transient; and wouldn't be available for records or further revision, so, the diagnosis depends only on the examiner's experience.

In the present study, five permanent staining techniques were used; Giemsa, ironhematoxylin, Masson's trichrome, Gram and modified acid-fast stains. The permanent stained smears provided a permanent record of the identified amoebae which can be used as a reference for consultations when unusual morphologic characteristics are found; sometimes because of many culture-related factors such as age, $\mathrm{pH}$, desiccation, and shortage of food (Walochnik et al, 2000; Garcia, 2007).

As regards staining quality, Giemsa stain, stained trophozoites blue and produced differences in intensity demonstrating the different organelles in the trophozoite stage. The nucleus stained darker inside the lighter colored vacuolated cytoplasm. The stain produced good contrast against background for both trophozoite and cyst stages. The Giemsa stain demonstrated dividing nuclei in Acanthamoeba trophozoite. The present findings were consistent with those of Abdul Majid et al. (2017) who used Giemsa stain for identification of FLA in treated and untreated water.

Considering Masson's trichrome stain, the results were consistent with Zeybek et al. (2010) and Ithoi et al. (2011) who used trichrome stain for characterization of FLA isolated from tap water samples and swimming pools respectively. The entire cell structures, including the nucleus were recognized easily in trophozoites of both Acanthamoeba and Naegleria. However, the nuclei of cysts of both genera weren't stained. Only the endocyst of Acanthamoeba was recognized with this stain. Also, the present results were concordant with Wannasan et al. (2013) who used trichrome stain for the morphological characterization of pathogen-ic FLA in flood water samples in Chiang Mai, Thailand, including Acanthamoeba and Naegleria. 
Using the iron hematoxylin stain, the cytoplasm, nucleus, food and water vacuoles of trophozoites were stained with a grey color only but were clearly differentiated from each other based on intensity variances. Acanthamoeba cysts had stained both outer and inner walls dark grey but without a visible nucleus, while the Naegleria cyst had a thin wall with a darker stained nucleus. The present results were more or less similar to those of Walsh (1986) and Ferreira (2003) who used iron hematoxylin stain for morphological identification of Entamoeba histolytica in stool.

Both modified acid-fast and Gram stains used in this study stained trophozoites blue and red respectively. But, the trophozoites' nuclei of Acanthamoeba and Naegleria were hardly recognized in contrast to the food and contractile vacuoles which were clearly seen in both genera. Regarding Acanthamoeba cyst, only the internal wall was deeplystained but the nuclei were not recognized. The nuclei of Naegleria cysts were hardly seen as well. These findings were consistent with those of Martinez and Visvesvara (1991) and da Rocha-Azevedo et al. (2009) who stated that Gram stain was not helpful for morphological characterization of amoebae as it did not clarify the characteristic nuclear morphology of amoebae different stages.

Regarding cyst stage, Giemsa, Gram and Masson's trichrome stains stained Acanthamoeba cyst's inner wall only. Meanwhile, modified acid-fast stain stained both inner and outer walls of Acanthamoeba cyst without a visualized nucleus. Giemsa, Gram, Masson's trichrome and modified acid-fast stains showed that Naegleria cyst had a thin wall and poorly recognized nucleus. In the present study, morphological observations agreed with Ithoi et al. (2011) who used Giemsa, Gram, iron hematoxylin, trichrome-eosin, and acid-fast stains to demonstrate the morphological characteristics of different developmental stages of Acanthamoeba and Naegleria.
Ithoi et al. (2011) reported that decolorizing was the most crucial step of the staining procedures. As an inadequate time for decolorizing resulted in a darker color hindering the visualization of the different cell organelles, while too much decolorization produced a lighter color and poor color contrast among the cell important structures.

In the present study, when comparing the time needed for finishing each permanent staining technique, the gram, the modified acid-fast and Giemsa stains took a relatively short time $(5,10 \& 20$ minutes respectively). But, iron hematoxylin and Masson's trichrome stains were time-consuming techniques, requiring approximately 60 minutes.

Considering the total procedure cost, Gram stain was the least expensive followed by modified acid-fast and Giemsa stains. Both iron hematoxylin and Masson's trichrome stains were more expensive. Establishing priorities in the process of assessment of different staining techniques vary extensively according to the purpose of the study and the available resources. Specifically, how important the attributes of staining quality, cost of stain, ease and time needed for processing are with respect to each other.

In view of the above observations, staining techniques were assessed using the method of multiattribute evaluation (MacPherson and McQueen, 1993). For epidemiological studies, each attribute is assumed of equal importance. The multi-attribute ranking showed the highest rank for Lugol's iodine and lactophenol cotton blue $(92.8 \%)$ while both iron hematoxylin and Masson's trichrome had the lowest rank (46.4\%). The present findings agreed with what reported previously by El-Sayed and Hikal (2015) study which showed the highest rank for temporary stains (iodine and eosin stains).

In the present study, Giemsa stain showed the second rank, being simple, fast, as well as more sensitive than the other permanent stains. These findings disagreed with ElSayed and Hikal (2015) who ranked Giemsa stain the least. However, the results agreed 
with Abdul Majid et al. (2017) who recommended the use of Giemsa stain for routine screening to identify the developmental stages of FLA in clinical, epidemiological and public health studies. Also, our results agree with those of Walochnik et al. (2015) who used Giemsa stained smears from corneal scrapings for diagnosis of AK and stated that this technique is the simplest and fastest diagnostic method. Also, our results agree with those of Zhang et al. (2018), who considered Giemsa the efficient stain to identify $N$. fowleri in CSF smears from cases of PAM

The present results agreed with that of Pirehma et al. (1999), Zeybek et al. ( 2010) and Younis et al. (2013). The authors found that although Masson's trichrome stain provides a proper sensitivity, this technique is complex and needs high laboratory skills and expertise, costly, time-consuming and was not suitable for survey study.

Iron-hematoxylin stain was used mainly in research, and not as a routine laboratory procedure because it is a too complex and time-consuming technique. The difficulty has been in the control of the decolorization step that requires good experience in the use of this stain (Ferreira, 2003).

Depending on the purpose, all these stains can be used to identify different stages of potentially pathogenic FLA for epidemiologic, clinical or even in public health use. In survey studies, the priority is for the fast, easy and cheap stain with respect to its diagnostic profit. The choice of the stain varies definitely in clinical situations where the detection is challenging due to the scarceness of the parasite. The priority here is for the quality of the staining technique.

\section{Conclusion}

Staining is a crucial step in the detection of different developmental stages of FLA to reveal the internal structures of trophozoites and cysts, which is the key for morphological identification by microscopy. Temporary staining using lactophenol cotton blue and/or Lugol's iodine is a simple technique that gives excellent fast results, with high quality and reasonable cost. Giemsa staining is consistently the most suitable among permanent staining techniques, yielding high-quality smears, with clear internal structural details. Also, the technique is simple, fast and needs no special microscopy or lab skills. In this regard, the combination of the wet mount and permanently stained smear techniques was recommended for routine and definitive diagnosis of FLA that would yield better results than does any single technique alone. Thus, for rapid, easy and efficient diagnosis of FLA by microscopy, wet mount staining, using lactophenol cotton blue or Lugol's iodine, should initially performed to be followed by Giemsa staining technique.

\section{Acknowledgment}

The present authors would like to present their thanks to Prof Dr Refaat MA Khalifa, Medical Parasitology Department Faculty of Medicine, Assiut University, Assiut, Egypt for revising the manuscript.

The authors wish to acknowledge Assiut University, Faculty of Medicine Grant Office, Assiut, Egypt, for the financial support to carry out this research work.

Ethical considerations: An ethical clearance was obtained from the Ethical Committee, Faculty of Medicine, Assiut University, approving the research protocol and its procedures (No: 17100773).

Conflict of interest: The authors confirmed that this manuscript is not under consideration for publication elsewhere and will not be submitted elsewhere. There are no conflicts of interest between the authors and any organization or between the authors themselves. They confirmed their responsible for the integrity and the accuracy of the data analysis in the study. There was no financial support of any kind for this research. We would also comply with any comments or suggestions.

\section{References}

Abdul Majid, MA, Mahboob, T, Mong, BGJ, Jaturas, N, Richard, RL, et al, 2017: Pathogenic waterborne free-living amoebae: An up- 
date from selected Southeast Asian Countries.PLoS One 8,12, 5:e0177564.

Balczun, C, Scheid, PL, 2017: Free-living amoebae as hosts for and vectors of intracellular microorganisms with public health significance. Viruses 9,65; doi:10.3390/v9040065.

Boggild, AK, Martin, DS, Lee, TY, Yu, B, Low, DE, 2009: Laboratory diagnosis of amoebic keratitis: comparison of four diagnostic methods for different types of clinical specimens. J. Clin. Microbiol. 47, 5:1314-18.

da Rocha-Azevedo, B, Tanowitz, HB, Marciano-Cabral, F, 2009: Diagnosis of infections caused by pathogenic free-living amoebae. Interdiscip. Perspect. Infect. Dis. doi: 10.1155/ 2009/251406.

El-Sayed, NM, Hikal, WM, 2015: Several staining techniques to enhance the visibility of Acanthamoeba cysts. Parasitol. Res. 114:823-30. Ferreira, CS, 2003: Staining of intestinal protozoa with Heidenhain's iron hematoxylin. Rev. Inst. Med. Trop. Sao Paulo 45(1):43-44.

Garcia, LS, 2007: Diagnostic Medical Parasitology ISM Press, Washington DC; Part II.

Grossniklaus, HE, Waring, GO, Akor, C, Castellano-Sanchez, AA, Bennett, K, 2003: Evaluation of hematoxylin and eosin and special stains for the detection of Acanthamoeba keratitis in penetrating keratoplasties. Am. J. Ophthalmol. 136, 3: 520-26.

Guarner, J, Bartlett, J, Shieh, WJ, Paddock, CD, Visvesvara, GS, et al, 2007: Histopathologic spectrum and immunohistochemical diagnosis of amebic meningoencephalitis. Mod. Pathol. 20, 12:1230-37.

Init, I, Lau, YL, Arin Fadzlun, A, Foead, AI, Neilson, RS, Nissapatorn, V, 2010: Detection of free-living amoebae, Acanthamoeba and Naegleria, in swimming pools, Malaysia.Trop. Biomed. 27: 566-77.

Ithoi, I, Ahmad, AF, Mak, JW, Nissapatorn, V, Lau, YL, Mahmud, R, 2011: Morphological characteristics of developmental stages of Acanthamoeba and Naegleria species before and after staining by various techniques. Southeast Asian J. Trop. Med. Publ. Hlth. 42:1327-38.

Khan, NA, 2006: Acanthamoeba: biology and increasing importance in human health. FEMS Microbiol. Rev. 30:564-95.

Khurana, S, Biswal, M, Kaur, H, Malhotra, P, Arora, P, et al, 2015: Free-living amoebae in water sources of critical units in a tertiary care hospital in India. Ind. J. of Med. Microbiol. 33:
343-48.

Król-Turmińska, K, Olender, A, 2017: Human infections caused by free-living amoebae. Ann. Agricul. Environ. Med. 24:254-60.

Le Govic, Y, Gonçalves, A, de Gentile, L, Pihet, M, 2018: Common staining techniques for highlighting Acanthamoeba cysts. Clin. Microbiol. Infect. 24, 9:970-1.

Macpherson, DW, Mcqueen, R, 1993: Cryptosporidiosis: multiattribute evaluation of six diagnostic methods. J. Clin. Microbiol. 31:198-202.

Martinez, AJ, Visvesvara, GS, 1991: Laboratory diagnosis of pathogenic free-living amoebas: Naegleria, Acanthamoeba, and Leptomyxid. Clin. Lab. Med. 11:861-72.

Martinez, AJ, Visvesvara, GS, 1997: Free-living, amphizoic and opportunistic amebas. Brain. Pathol. 7, 1:583-98.

Ozcelik, S, Coskun, KA, Yunlu, O, Alim, A, Malatyali, E, 2012: The prevalence, isolation, and morphotyping of potentially pathogenic free-living amoebae from tap water and environmental water sources in Sivas. Turk. Parazitol. Dergisi 36:198-203.

Page, FC, 1988: A new key to freshwater and soil gymnamoebae: with instructions for culture, Freshwater Biological Association.

Pirehma, M, Suresh, K, Sivanandam, S, Anuar, KA, Ramakrishnan, K, et al, 1999: Field's stain: A rapid staining method for Acanthamoeba spp. Parasitol. Res. 85:791-93.

Schuster, FL, Visvesvara, GS, 2004: Free-living amoebae as opportunistic and non-opportunistic pathogens of humans and animals. Int. J. Parasitol. 34: 1001-27.

Sharma, S, Grag, P, Roa, GN, 2000: Patient characteristics, diagnosis and treatment of noncontact lens related Acanthamoeba keratitis. Br. J. Ophthalmol. 84, 10:1103-8.

Shen, J, Jiang, QW, Li, QX, Chen, HY, Li, Z H, 2005: Gimenez staining: a rapid method for initial identification of legionella pneumophila in Amoeba trophozoite. Zhongguo Ji Sheng Chong Xue Yu Ji Sheng Chong Bing Za Zhi 23, 4: 240-2.

Teixera, LH, Rocha, S, Pinto, RMF, Caseiro, MM, da Costa, SOP, 2009: Prevalence of potentially free-living amoebae from Acanthamo$e b a$ and Naegleria genera in non-hospital, public, interval environments from the city of Santos, Brazil. Braz. J. of Infect. Dis. 13: 395-97.

Thomas, PA, Kuriakose, T, 1990: Rapid detection of Acanthamoeba cysts in corneal scrap- 
ings by lactophenol cotton blue staining. Arch. Ophthalmol. 108, 2:168-70.

Thomas, PA, Jesudasan, CAN, Kaliamurthy, J, 2011: Rapid detection of Acanthamoeba cysts in corneal scrapings by chlorazol black E staining. Can. J. Ophthalmol. 46, 5:443-4.

Visvesvara, GS, 1999: Pathogenic and opportunistic free-living amoebae. In: Murray PR, Baron EJ, Pfaller MA, Tenover FC, Yolken RH, ed. Manual of clinical microbiology. $7^{\text {th }}$ ed. Washington DC: ASM Press.

Visvesvara, GS, Moura, H, Schuster, FL, 2007: Pathogenic and opportunistic free-living amoebae: Acanthamoeba spp., Balamuthia mandrillaris, Naegleria fowleri and Sappinia diploidea. FEMS Immunol. Med. Microbiol. 50:1-26.

Walochnik, J, Obwaller, A, Aspock, H, 2000: Correlations between morphological, molecular biological and physiological characteristics in clinical and nonclinical isolates of Acanthamoeba spp. Appl. Environ. Microbiol. 66:4408-13. Walochnik, J, Scheikl, U, Haller-Schober, E M, 2015: Twenty years of Acanthamoeba diagnostics in Austria. J. Eukaryot. Microbiol. 62, 1: 3-11.

Walsh, JA, 1986: Problems in recognition and diagnosis of amebiasis: estimation of the global magnitude of morbidity and mortality. Rev. Infect. Dis. 8, 2:228-38.

Wannasan, A, Uparanukraw, P, Songsangchun, A, Morakote, N, 2013: Potentially pathogenic free-living amoebae in some flood-affected areas during 2011 Chiang Mai flood. Rev. Inst. Med. Trop. Sao Paulo 55, 6:411-16.

Year, H, Zamfir, O, Bourcier, T, Ancelle, T, Batellier, L, et al, 2007: Comparison of PCR, microscopic examination and culture for the early diagnosis and characterization of Acanthamoeba isolates from ocular infections. Eur. J Clin. Microbiol. Infect. Dis. 26:221-4.

Younis, MS, Elhamshary, AM, Abd-Elmaboud, AI, El-Sayed, NM, Kishik, SM, 2013: Diagnosis of Acanthamoeba keratitis in clinically suspected cases and its correlation with some risk factors. Egy. J. Med. Sci. 34, 2:527-40.

Zhang, LL, Wu, M, Hu, BC, Chen, HL, Pan, JR, et al, 2018: Identification and molecular typing of Naegleria fowleri from a patient with primary amebic meningoencephalitis in China. Int. J. Infect. Dis. 72:28-33.

Zeybek, Z, Miray Üstüntürk, M, Binay, AR, 2010: Morphological characteristics and growth abilities of free-living amoeba isolated from domestic tap water samples in İstanbul. Eur. J. Biol. Arch. 69, 1:17-23.

\section{Explanation of figures}

Fig.1: Acanthamoeba and Naegleria trophozoites and cysts stained with Lugol's iodine stain (a) Acanthamoeba trophozoite showing characteristic acanthopodia (arrow) with a single nucleus (arrowhead), (b) Acanthamoeba cyst showing an outer wall "ectocyst" (long arrow), inner wall "endocyst" (short arrow), with a single nucleus (arrowhead), (c) Naegleria trophozoite showing blunt hemispherical bulge (lobopodium) at the anterior end (arrow) and a single nucleus (arrowhead), (d) Naegleria cyst showing a single nucleus (arrowhead) (x1000).

Fig.2: Acanthamoeba and Naegleria trophozoites and cysts stained with lactophenol cotton blue stain (a) Acanthamoeba trophozoite showing characteristic acanthopodia (arrow) with a single nucleus (arrowhead), (b) Acanthamoeba cyst showing the ectocyst (long arrow) and the endocyst (short arrow) with a single nucleus (arrowhead), (c) Naegleria trophozoite showing blunt hemispherical bulge (lobopodium) at the anterior end (arrow) and a single nucleus (arrowhead), (d) Naegleria smooth spherical cyst with a single nucleus (arrowhead) (x1000).

Fig.3: Acanthamoeba and Naegleria trophozoites and cysts stained with Giemsa stain (a) Acanthamoeba trophozoite showing characteristic acanthopodia (arrows) and a single nucleus (arrowhead) with highly vacuolar cytoplasm, (b) Acanthamoeba trophozoite showing two nuclei (arrowheads, (c) Acanthamoeba cysts showing unstained ectocyst and well-stained endocyst, (d) Naegleria trophozoite showing blunt lobopodium at the anterior end (arrow) with a single nucleus (arrowhead), (e) Naegleria cyst with a single nucleus (arrowhead) (x1000).

Fig.4: Acanthamoeba and Naegleria trophozoites and cysts stained with iron hematoxylin stain (a) Acanthamoeba trophozoite showing characteristic acanthopodia (arrow), with a single nucleus (arrowhead) and highly vacuolar cytoplasm, (b) Acanthamoeba cysts showing the ectocyst (arrowheads) and the endocyst (arrows), (c) Naegleria trophozoite showing blunt hemispherical bulge (lobopodium) at the anterior end (arrow) and a single nucleus (arrowhead), (d) Naegleria cyst showing single nucleus (arrowhead) (x1000).

Fig.5: Acanthamoeba and Naegleria trophozoites and cysts stained with Masson's trichrome stain (a) Acanthamoeba trophozoites showing characteristic acanthopodia (arrows), with a single nucleus (arrowheads) and highly vacuolar cytoplasm, (b) Acanthamoeba cyst showing unstained ectocysts (arrow) and well stained endocysts (arrowhead), (c) Naegleria trophozoite showing a single nucleus (arrowhead) and vacuolar cytoplasm, (d) Naegleria smooth spherical cyst (x1000).

Fig.6: Acanthamoeba and Naegleria trophozoites and cysts stained with modified acid-fast stain (a) Acanthamoeba trophozoite showing characteristic acanthopodia (arrowhead) with highly vacuolar cytoplasm, (b) Acanthamoeba trophozoite showing single nucleus (arrowhead), (c) Acanthamoeba cyst showing the ectocyst (arrowhead), and the endocyst (arrow), (d) Naegleria trophozoite showing blunt hemispherical bulge (lobopodium) at the anterior end (arrow) and a single nucleus (arrowhead), (e) Naegleria smooth spherical cyst with a single nucleus (arrowhead) (x1000).

Fig.7: Acanthamoeba and Naegleria trophozoites and cysts stained with Gram stain (a) Acanthamoeba trophozoite showing a single nucleus (arrowhead) and highly vacuolar cytoplasm, (b) Acanthamoeba cyst showing well-stained endocyst and unstained ectocyst, (c) Naegleria trophozoite showing blunt hemispherical bulge (lobopodium) at the anterior end (arrow), a single nucleus (arrowhead) and prominent contractile vacuole at the posterior end (star), (d) Naegleria cyst showing single nucleus (arrowhead) (x1000). 


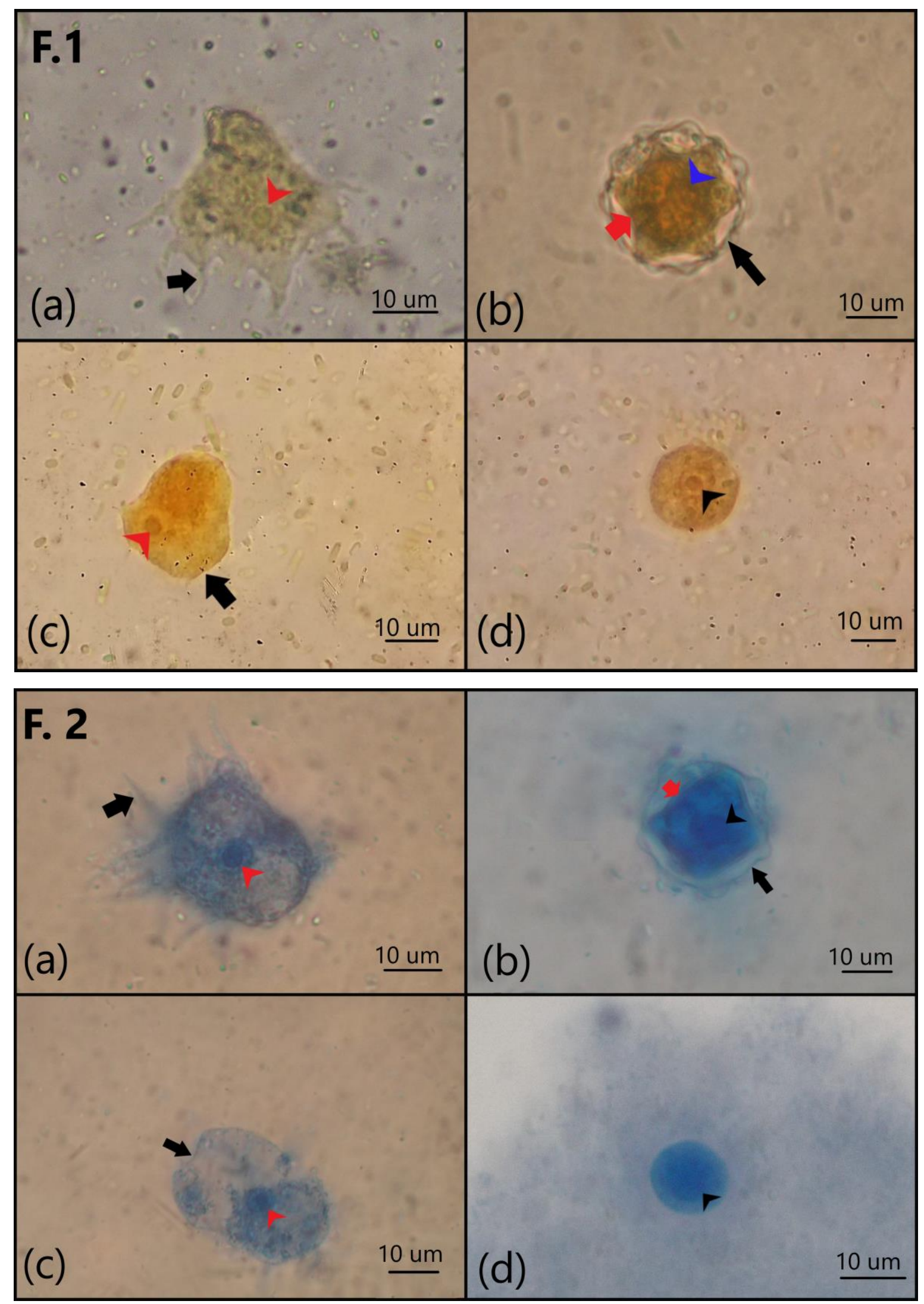



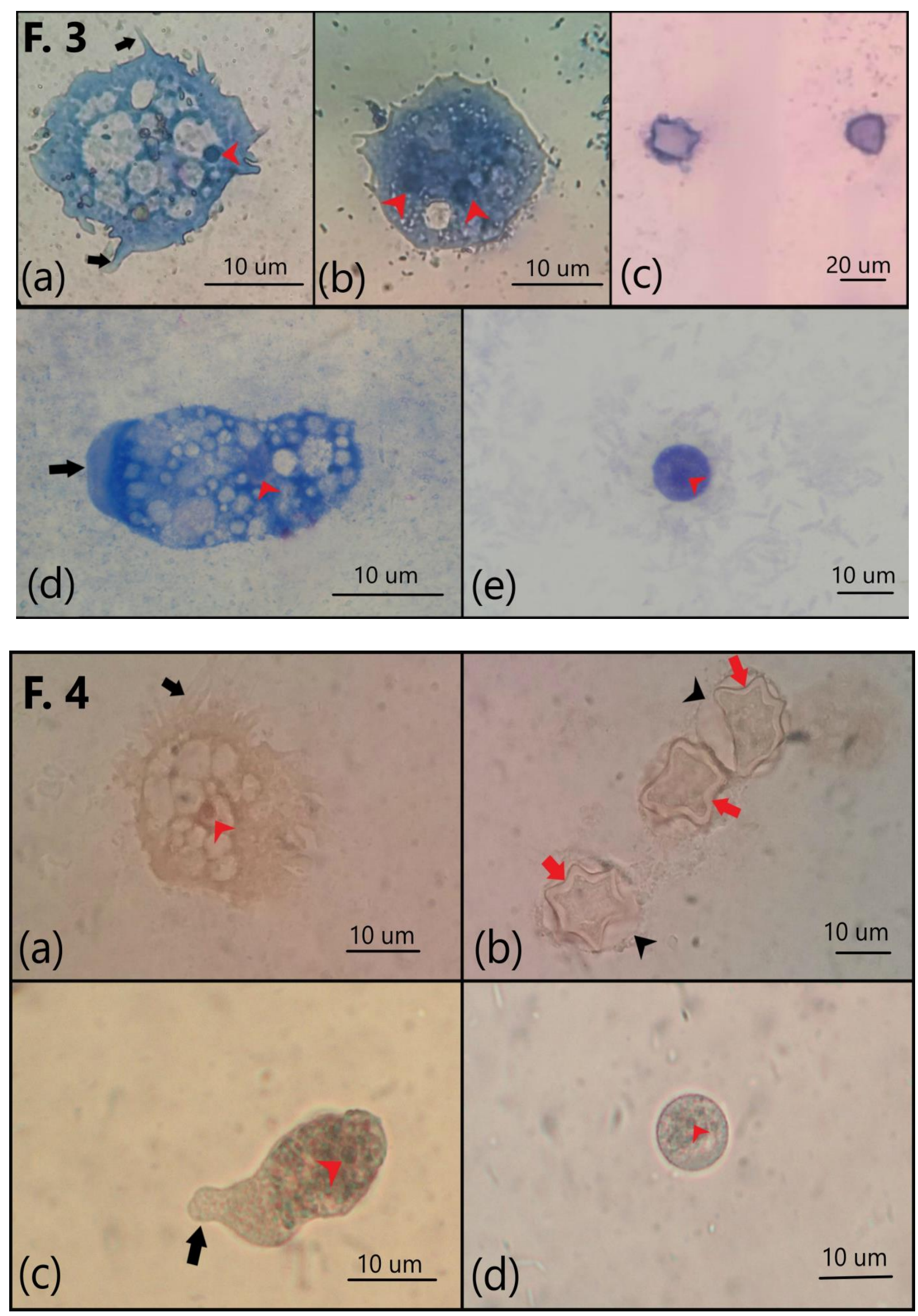

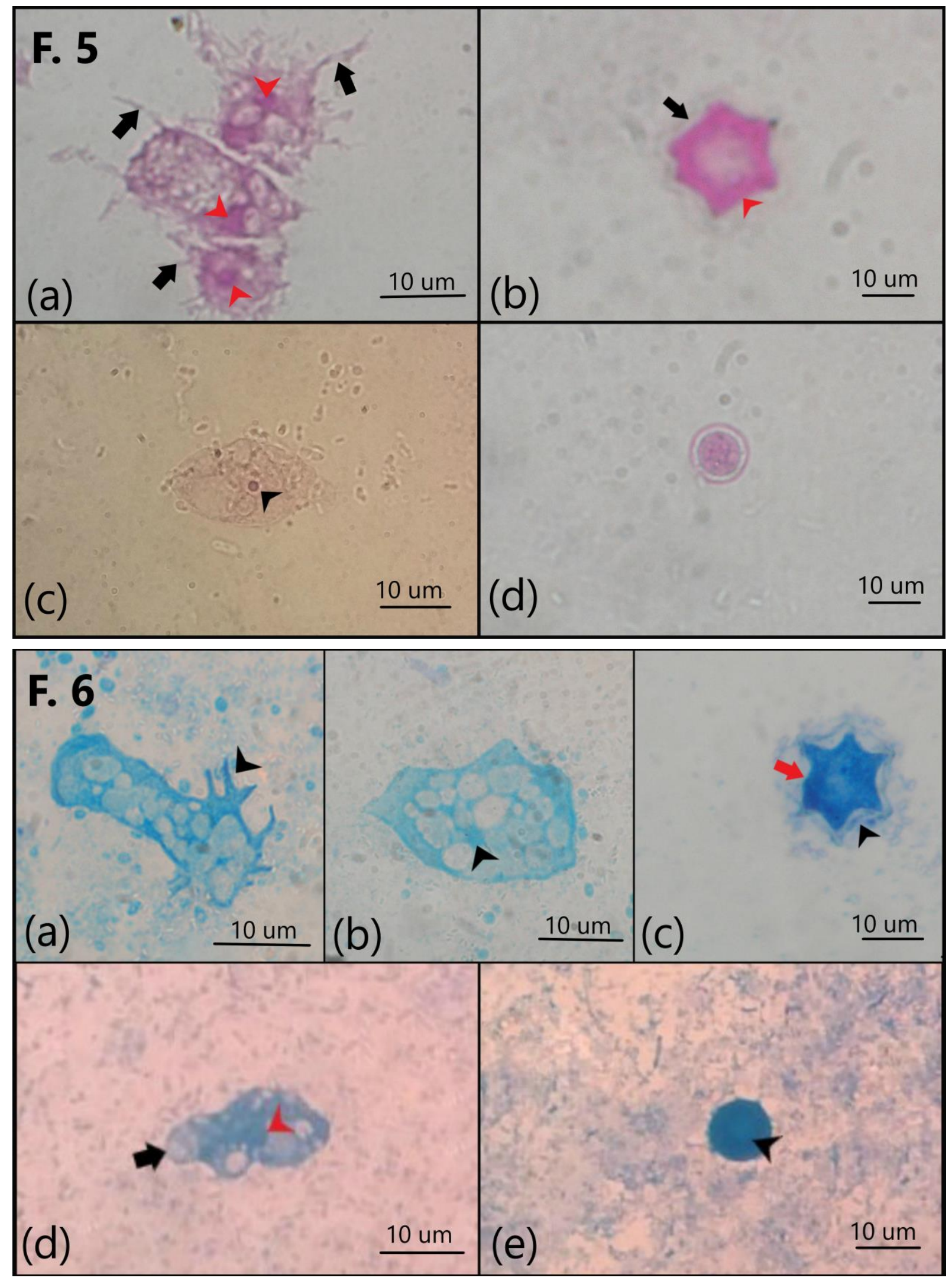


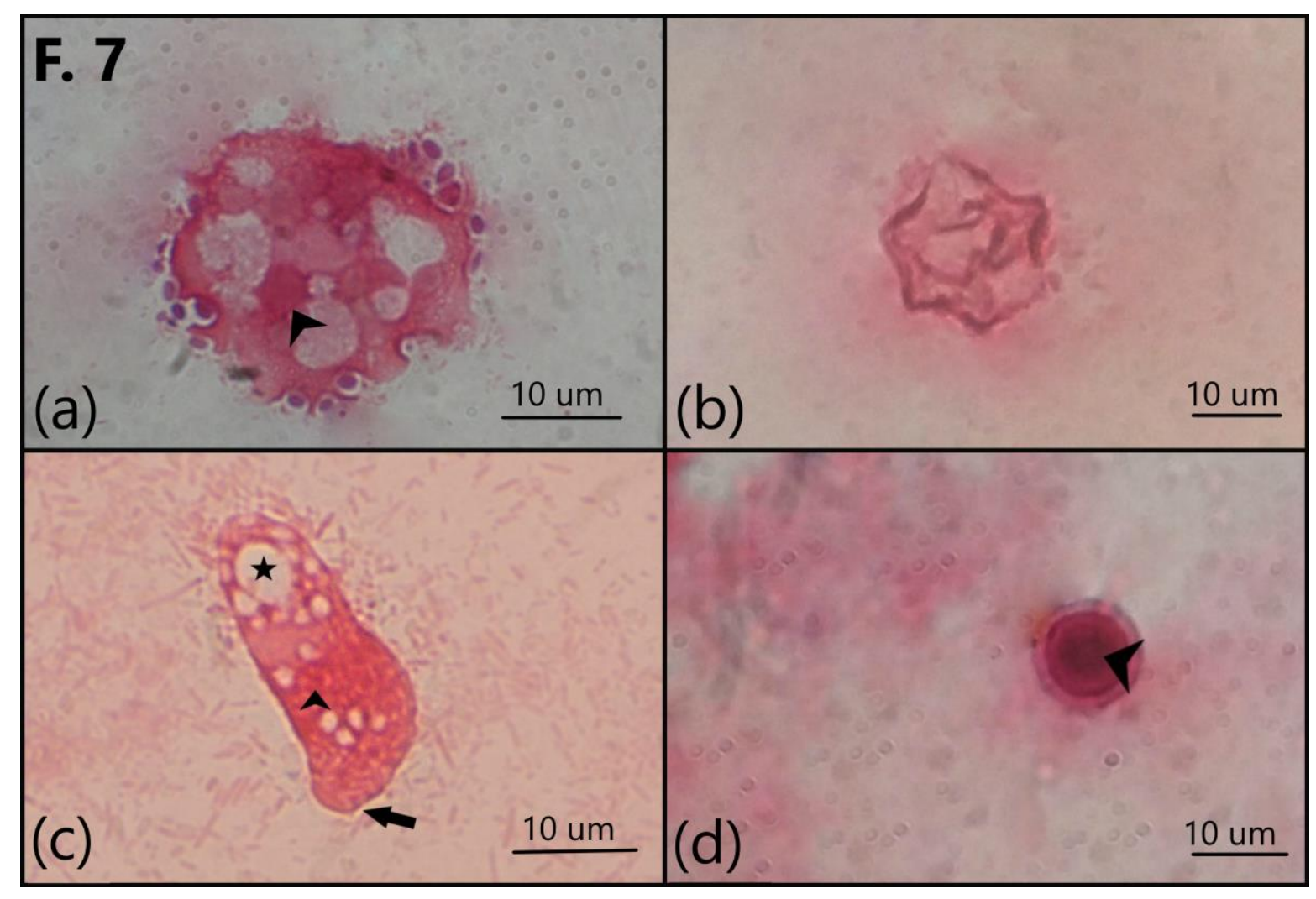

